\title{
EXPOSITION, REPRÉSENTATION ET COMMUNICATION
}

\author{
Jean Davallon'1 et Joëlle Le Marec ${ }^{2}$
}

La confrontation et la discussion de deux études menées sur les représentations liées à des thématiques de l'environnement ont été l'occasion de questionner l'apport de l'étude des représentations sociales à la conception des expositions de sciences et de techniques. La première de ces études a été effectuée dans le cadre de la conception d'une exposition sur le thème de l'environnement à la Cité des sciences et de l'industrie; la seconde, dans le cadre de la programmation d'un musée sur la chimie ${ }^{3}$.

1 Professeur et directeur du Centre d'étude et de recherche sur les expositions et les musées (CEREM), Université Jean Monnet, Saint-Etienne.

2 Responsable de la cellule Évaluation de la direction des Expositions, Cité des sciences et de l'industrie, Paris, et Centre d'étude et de recherche sur les expositions et les musées (CEREM), Université Jean Monnet, Saint-Etienne.

${ }^{3}$ Il s'agit respectivement d'une étude dirigée par Joëlle Le Marec (L'Homme et son environnement, Paris, Direction des expositions- Cité des sciences et de l'industrie, 1990) et du second volet de l'étude dirigée par Jean Davallon (Étude en vue de l'élaboration d'un Cahier de Programmation muséale, Lyon/Saint-Fons: LARMURAL/Ville de Saint-Fons, 1991); second volet intitulé : 2. Étude détaillée des représentations de la chimie (Jean Davallon, Emmanuelle François, avec la coll. de Aurélie Lanquetot). Dans la mesure où, à partir de ces deux enquêtes, cet article développe une réflexion de recherche, nous nous permettons de faire référence à un certain nombre de nos propres travaux, ou de travaux de l'équipe, sur lesquels il s'appuie et qu'il prolonge.

Recherches en communication, $n^{\circ} 4,(1995)$. 
Au-delà du projet d'optimiser la communication avec le visiteur, qui caractérise toute évaluation préalable, c'est à une interrogation sur le fonctionnement de la mise en communication des savoirs dans l'exposition que l'on aboutit.

\section{Deux études sur les représentations liées à l'environnement}

Les deux études choisies présentent l'intérêt de posséder simultanément deux particularités. D'un côté, elles s'inscrivent dans le projet d'une optimisation de la conception de l'exposition. D'un autre côté, le thème de ces expositions est investi d'une forte charge symbolique. Ainsi, la conjonction d'un objectif qui vise à faire de l'exposition un outil de communication des savoirs scientifiques plus performant, avec un thème impliquant fortement les sujets sociaux a certainement contribué à favoriser une analyse de la complexité des systèmes de représentations ${ }^{1}$. Cela a obligé à approfondir la technique d'enquête; ce qui a eu pour conséquence de faire apparaître plus clairement qu'à l'accoutumée la place des représentations des visiteurs et, au bout du compte, de mieux comprendre ce que signifiait communiquer par l'exposition.

C'est la compréhension de cette double particularité -et surtout de ses conséquences- qui justifie une discussion quelque peu détaillée de ces deux études.

\section{L'évaluation préalable au service de la communication par l'exposition}

Dans la mesure où le visiteur devient le centre du discours muséal contemporain, l'exposition est souvent vue comme "un moyen terme entre l'inconnu du savoir savant (des arts et des sciences), et le déjà-là du savoir profane. L'objectif de la démarche évaluative étant de faire en sorte que le discours savant rencontre le déjà-là des

1 J. Davallon, G. Grandmont, B. SChiEle, L'environnement entre au musée. Lyon, Presses universitaires de Lyon, coll. Muséologies, Québec, Musée de la civilisation à Québec, 1992. Trad. angl.: The Rise of Environmentalism in Museums, Québec, Musée de la civilisation. 
représentations du profane pour mieux le dépasser"1. Cet objectif communicationnel de l'exposition, un concepteur de la Cité des sciences et de l'industrie le formulait différemment, lors d'une séance de travail, d'une manière encore plus "intentionnelle": "l'exposition scientifique et technique a pour objectif de réduire l'écart entre la façon dont les scientifiques et les visiteurs se posent des questions". L'évaluation préalable est issue de ces préoccupations. Son rôle, tel qu'il a été défini dans le modèle de l'évaluation comme outil au service de la conception, qui domine depuis près de trente ans, en particulier dans les musées des sciences et des techniques, est d'anticiper les réactions du visiteur à ce qui sera présenté afin de prévoir des dispositifs qui l'amèneront à dépasser son déjà-là, pour faire un maximum de chemin en direction du savoir scientifique transmis par l'exposition. C'est en cela qu'il s'inscrit dans une démarche d'optimisation de la conception, se situant dans le droit fil de l'ensemble des idées qui ont conduit à la définition d'un cycle d'évaluation selon trois phases avant/après la production de l'exposition : évaluation préalable, formative et sommative ${ }^{2}$.

Au sein des études préalables, au cours des dernières années, s'est développé l'étude des représentations de visiteur sur un thème donné ${ }^{3}$. Ce courant des études de représentations se démarque des évaluations préalables habituellement menées dans le domaine des expositions de sciences et techniques, visant essentiellement à mesurer l'écart entre un savoir de sens commun et une connaissance scientifique. Il se démarque également des enquêtes portant sur les opinions ou les attitudes. Il se donne en effet comme objectif

1 B. SCHIELE, "L'invention simultanée du visiteur et de l'exposition", Publics \& musées, 2, 2ème sem., p. 71-98.

2 Pour plus de détails, on pourra se reporter à la synthèse d'Hana Gottesdiener (Évaluer l'exposition: définitions, méthodes et bibliographie sélective commentée d'études d'évaluation, Paris, La Documentation française, 1987) ou à la présentation des pratiques d'évaluation de $\mathrm{H}$. Shettel et St. Bitgood ("Les pratiques de l'évaluation des expositions", Publics \& musées, 4, mai 1994, p. 9-25).

3 Les évaluations préalables menées au sein de la direction des expositions à la Cité des sciences et de l'industrie depuis 1989 (J. LE MAREC, "Les évaluations préalables: une aide à la conception des expositions", La Lettre de l'Office de coopération et d'information muséographiques (OCIM), 22, 1992, p. 21-26) ont fortement contribué au développement de ce type d'études. Parallèlement, des recherches se référant à l'analyse des représentations élaborée par Moscovici ont été menées au Québec et en France dans le contexte des études sur la vulgarisation des savoirs scientifiques. L'étude pour le musée de la chimie s'inscrivait dans cette référence à Moscovici. 
d'appréhender les cadres de référence à partir desquels les visiteurs aborderont l'exposition; cadres qui sont à concevoir comme une "compétence" (au sens que les linguistes donnent à ce terme) que le visiteur mobilisera lorsqu'il sera engagé dans la compréhension de tout ce qui sera présenté dans l'exposition.

Mais afin d'ouvrir une discussion de ces caractéristiques des études de représentation qui ne nous éloigne pas de notre propos, nous proposons de commencer par un point rapide sur les résultats obtenus dans les deux études. Le phénomène marquant -qui est d'ailleurs à l'origine de la présente réflexion- est la proximité des types de réponses obtenus dans chacune des deux études, tant du point de vue des images mobilisées, du mode d'implication des personnes interrogées que du rapport entre science et société.

\section{Des images mobilisées fortement structurées}

Viennent en premier des images suscitées par l'évocation du thème: "chimie", ou bien "environnement". Ces images n'illustrent pas à proprement parler un thème (ces deux thèmes ne sont pas des objets que l'on se représente simplement); elles sont plutôt des sortes de condensations "consistantes" de l'ensemble des idées, sentiments, références, que recouvre le thème pour les personnes interrogées.

Par exemple, dans le cas de la chimie, ces images peuvent être regroupées selon cinq grandes catégories : la formule/le symbole, le laboratoire/l'usine, les produits chimiques, les catastrophes/la pollution, l'alchimiste/le chimiste fou. L'important dans ces images est leur connotation clairement négative : elles renvoient à la crainte du danger que génère l'activité chimique mais aussi à l'expérience scolaire qui a été vécue négativement, liée à un fort sentiment d'incompréhension. On s'aperçoit ainsi que l'expérience scolaire laisse des traces beaucoup plus fondamentales et "structurantes" que ce qu' on en aurait retrouvé par le biais de la recherche de conceptions naïves en chimie.

Dans le cas de l'environnement, ce qui est évoqué prend trois formes possibles. Soit il s'agit de la nature vue comme un grand tout tel qu'évoqué selon un tableau construit à la manière d'un inventaire “infini”: les éléments, l'air, l'eau, l'atmosphère, les lacs, les rivières, les fleurs, la campagne, la montagne, les forêts, les sources, etc. Soit on a affaire à des références directes au cadre de vie quotidien (ville, 
habitat par exemple). Soit ce sont les problèmes environnementaux qui sont visualisés par certaines figures-clés: par exemple, le problème de la couche d'ozone -abondamment commenté par les visiteurs alors même qu'il n'est pas considéré par eux comme préoccupant- semble concrétiser pour eux ce qu'est un problème d'environnement, en fournir le modèle. La couche d'ozone est une enveloppe protectrice autour de la terre, enveloppe dont l'intégrité a été violée par l'homme. À cause de cette faute, de cette fausse mancuvre, l'enveloppe est déchirée et laisse passer ce qui est dangereux, le mal. Une telle modélisation fonctionnelle de la situation se passe fort bien d'explications scientifiques: interrogés sur le processus d'altération de la couche d'ozone, les visiteurs peuvent évoquer le réchauffement de l'atmosphère par le $\mathrm{CO}^{2}$. Par la fissure peuvent passer toutes sortes d'émanations, rayonnements, radiations, pollutions. Peu importe, à vrai dire, le nom de ces émanations, car l'important est qu'elles sont nocives.

On le voit, ce qui est essentiel dans ces réponses, c'est que le savoir scientifique n'a pratiquement aucune pertinence pour le visiteur: pour lui, le "vrai" processus se déroule au niveau non pas du phénomène, tel qu'il peut être analysé par les scientifiques, mais à celui plus "opérationnel" des relations entre l'homme et la nature: chacune des images offre une figure des effets que produit la société humaine.

\section{Une implication des personnes interrogées}

Dans les deux études, au-delà des premières images, les personnes interrogées sont confrontées à l'invisibilité essentielle de ce que recouvrent réellement la chimie ou les problèmes d'environnement. Cette invisibilité est le fait d'un savoir progressivement mis à distance parce que resté opaque, incompréhensible: une invisibilité qui s'enracine dans une expérience négative de ne rien comprendre à la science et qui est fortement liée, dans le cas de la chimie, à l'expérience scolaire.

Mais les représentations s'ancrent encore plus profondément: l'invisibilité est aussi la marque d'une puissance mal maîtrisée et par conséquent dangereuse. Ainsi, dans le cas de la chimie, seuls des effets sont visibles, les causes restent quant à elles des processus invisibles et incompréhensibles. Dans les deux études, chimie et 
environnement, le sentiment d'incompréhension renvoie à l'idée qu'il s'agit là de phénomènes qui échappent à la maîtrise de l'homme, qui relèvent d'une puissance invisible. Ainsi, en est-il de la pollution: "la pollution, on vit avec, on ne la voit pas, on ne se rend pas vraiment compte". Il arrive d'ailleurs que le visiteur cite, en même temps qu'un problème d'environnement grave, son propre problème de maîtrise d'une situation difficile à percevoir.

Plus profondément encore, on s'aperçoit, lorsque l'on pousse l'analyse, que le sentiment de ne pouvoir maîtriser cette puissance invisible se fonde sur un système d'oppositions de nature mythique qui engage les relations de l'homme avec la nature. Dans le cas de la chimie, c'est la transformation de la matière (la réaction chimique provoquée) qui risque de rompre un ordre de la nature conçu comme un équilibre achevé et harmonieux. De nombreuses autres études préalables réalisées à la Cité des sciences et de l'industrie sur les thèmes de la santé, du littoral, de l'espace, de l'agriculture, font apparaître continuellement ce système d'opposition, dans lequel l'accent est mis tantôt sur la nature en danger, tantôt sur la logique purement humaine du progrès technique destructeur, antinomique de l'ordre naturel. C'est le sentiment d'une conjonction entre d'une part la puissance quasi illimitée de la technique et du progrès, et d'autre part la perte de la maîtrise des effets de cette puissance, qui génère un faisceau d'attitudes très ambivalentes à l'égard des sciences et techniques, et un sentiment d'anxiété profonde.

\section{Une dimension sociale des savoirs}

Avec de tels thèmes, qui impliquent profondément le visiteur, les sciences semblent avant tout être perçues comme un des secteurs de l'activité humaine. La science peut être vue non pas comme le champ autonome de la construction du savoir, mais au contraire, comme une activité par trop subordonnée aux intérêts de l'industrie et du progrès technique. Pour les visiteurs, ce qui est important, ce qui semble le plus pertinent n'est pas une meilleure connaissance des phénomènes ou des techniques. D'une façon assez nette, il apparaît que c'est sur la maîtrise, sur le contrôle social, politique et éthique des activités humaines et de la fatale logique du progrès, que l'on doit miser avant tout. 
Les attentes formulées par les visiteurs vis-à-vis du projet d'exposition sur l'environnement ou du musée de la chimie sont très cohérentes avec le type d'expérience qu'ils entretiennent avec le thème.

Par exemple, les personnes qui ont travaillé dans l'industrie chimique à Saint-Fons attendent que le musée soit un lieu d'inscription de la mémoire de leur expérience de travail. Celles qui ont une expérience de la chimie comme savoir, ont des attentes tournées vers la vulgarisation, de la matérialisation de données abstraites. À un autre niveau, les futurs visiteurs de l'exposition sur l'environnement souhaitent voir une présentation des effets "avant/après" (la forêt avant et après les pluies acides par exemple), des comparaisons entre des états différents de l'environnement. L'exposition est vue comme étant le moyen de visualiser des phénomènes, de les toucher du doigt.

Enfin, les personnes ayant la simple expérience de la chimie ou de l'environnement liée à une utilisation quotidienne émettent une forte revendication d'information "sur" le savoir scientifique (et non pas une attente de savoir à la manière des scientifiques). L'attente est alors orientée vers le contexte social ou les implications individuelles de la chimie, ou encore vers l'engagement collectif global dans une action en faveur de l'environnement. En ces cas, les personnes interrogées souhaitent que soient rendus visibles "le schéma social" définissant les responsabilités de chacun (État, industriel, citoyens...) ainsi que les activités et savoirs qui sont impliqués dans ce schéma social: "savoir où on en est" ou à tout le moins, "se faire une idée", pour savoir ce qu'il convient de faire. Cette attitude sous-entend une demande de reconnaissance, une revendication de participation au concert social. On demande que le progrès ne se fasse pas sans transparence, et que chaque visiteur puisse être considéré en tant que citoyen.

Faut-il préciser que les quelques résultats que nous venons de présenter nous intéressent moins ici en eux-mêmes que pour le décalage qu'ils indiquent entre une étude des visiteurs centrée sur le contenu scientifique et une approche en termes de représentations sociales... 


\section{Les représentations, indicateur d'une nouvelle approche de la communication par l'exposition}

Tous les professionnels des musées sont à peu près d'accord avec l'idée que le visiteur arrive avec des représentations, sorte de bruit de fond informe, continuellement présent avec lequel il faut compter et dont on peut extraire, pour mieux les connaître et les prendre en compte, des "particules" précises. Or, ce que nous voyons se dessiner dans l'étude préalable de ces représentations présentée à l'instant, c'est la forme de ces représentations, la puissance et la cohérence de leur architecture, à la charnière entre la dimension sociale et la dimension cognitive de la construction des savoirs. Il ne s'agit plus d'un savoir par bribes, morcelé, truffé d'erreurs, d'opinions (un savoir en somme "amorphe"), mais bien d'un savoir socialisé et intégrateur. Arrêtons-nous sur cette différence.

\section{Le fonctionnement des représentations sociales}

Lorsqu'on parle des "représentations", on a habituellement à l'esprit des thèmes, des idées, des contenus qui seraient présents dans la tête des gens. Par exemple: se demander comment les gens se représentent l'environnement revient alors à essayer de savoir quelle idée ils s'en font. Si nous admettons que les représentations sont des cadres et constituent une compétence, une telle définition ne saurait suffire. Elles ne peuvent être simplement posées comme des contenus opposés à d'autres contenus que seraient les savoirs présentés dans l'exposition.

Il faut au contraire se rapprocher d'une conception de la représentation du type de celle proposée par Serge Moscovici sous le terme de "représentation sociale": l'entendre comme un processus à la charnière du social, de l'affectif et du cognitif qui forme les cadres à partir desquels nous appréhendons -percevons et comprenons- la réalitél. Faisant le point sur la question dans le cadre d'une présentation

1 Ce concept est élaboré et opérationnalisé pour la première fois par S. Moscovici (La psychanalyse, son image et son public, Paris, P.U.F., 1976 [1961]). On trouvera une présentation de la question dans D. Jodelet ("Représentation sociale: phénomènes, concept et théorie", in S. MosCovicI (Dir.), Psychologie sociale, Paris, P.U.F., coll. Fondamental, 1984, p. 357-378 et Les représentations sociales, Paris, P.U.F., 1989). 
des concepts de la psychologie sociale, Gustave-Nicolas Fischer ${ }^{1}$ définit la représentation sociale comme "un processus d'élaboration perceptive et mentale de la réalité qui transforme les objets sociaux (personnes, contextes, situations) en catégories symboliques (valeurs, croyances, idéologies) et leur confère un statut cognitif permettant d'appréhender les aspects de la vie ordinaire par un recadrage de nos propres conduites à l'intérieur des interactions sociales".

Dans le cas qui nous occupe, cette approche des représentations sociales présente l'immense avantage de fournir un modèle effectif d'approche du "déjà-là" des visiteurs. Cette théorie apporte en effet un double éclairage sur ce que disent les personnes interrogées; double éclairage qui correspond à la double fonction des représentations sociales: fonction d'objectivation et fonction d'ancrage. Rappelons que la fonction d'objectivation est le processus par lequel des données abstraites ou conceptuelles deviennent des images concrètes. Il est admis que ce processus de traitement des savoirs s'appuie sur trois opérations : le tri des informations (filtrage), la condensation des éléments d'informations en grandes figures ("noyau figuratif" issu d'une opération de schématisation structurante), et enfin la transformation des informations retenues en réalités évidentes (les concepts tendent à devenir des choses par naturalisation). La fonction d'ancrage est le processus par lequel les représentations $s$ 'insèrent dans le social, la manière dont elles constituent des cadres d'interprétation de ce que l'on voit et vit. Elles participent aux systèmes de valeur de la société et contribuent en retour à la formation de nouvelles formes de pensée.

Ainsi, en nous référant à la fonction d'objectivation, on peut considérer ce que nous avons désigné plus haut comme "les images", comme la partie la plus manifeste des représentations, celle qui apparaît en premier. Ces images correspondent à la partie la plus cristallisée des représentations sous la forme de "figures" 2 Mais tandis que les approches qui s'intéressent principalement au contenu des représentations valorisent la dimension des images de manière presque exclusive, la prise en compte de la fonction d'ancrage va conduire à s'interroger sur ce qui donne sa logique à ces représentations, aux

1 G.-N. FISCHER, Les concepts fondamentaux de la psychologie sociale, ParisMontréal, Dunod-Presses de l'Université de Montréal, 1991, p. 118.

2 On trouve une analyse de ces processus d'objectivation appliquée à la différence entre science et sens commun dans S. MoscoviCI et M. HEWSTONE, "De la science au sens commun", in S. Moscovici (Dir.), Psychologie sociale, op. cit., p. 539-566. 
façons de penser dans lesquelles celles-ci s'insèrent et à la manière dont les gens se les approprient. Il faut alors chercher comment ces représentations font système. Plus précisément encore, comment elles intègrent les objets, thèmes et discours des expositions dans des systèmes d'oppositions symboliques qui sont au fondement anthropologique de notre culture (au sens de notre "vision du monde"). C'est ainsi par exemple que l'on découvre que le thème de "l'environnement" renvoie à des oppositions fondamentales (anthropologiquement s'entend) telle que nature vs culture, vie vs mort, soi vs autre.

Sous cet angle, une telle opposition qui à bien des égards pouvait paraître banale, compte tenu de sa généralité, prend pour l'évaluation des expositions un relief nouveau. Elle lui pose en effet la question de la relation entre sens et savoirs, bien plus que celle d'une appropriation efficace des savoirs. La théorie des représentations sociales fournit ainsi un cadre anthropologique dans lequel replacer ce qui est dit par les personnes interrogées. Au fond, c'est un changement du statut du discours tenu par ces dernières qui est ainsi en fait engagé.

\section{Conséquences sur le statut du discours tenu par les personnes interrogées}

Le discours des personnes interrogées possède une cohérence et ne relève ni de l'association libre ni de l'influence mécanique des médias. Comme toutes choses sociales, les représentations que l'on y trouve possèdent un fonctionnement autrement plus fin et plus complexe.

Reprenons. Dans les deux études présentées, le système des représentations met en jeu trois niveaux. Le premier, à la fois le plus évident, figuré et immédiat, correspond, nous l'avons vu, aux images. À l'opposé, le niveau le plus "profond" est constitué d'oppositions (du type homme vs nature) appartenant à des catégories symboliques stables et générales. Rappelons que les images sont repérables en clair dans le discours recueilli lors des entretiens, tandis que les catégories résultent d'un travail d'analyse, elles sont des construits. Entre ces deux niveaux, en existe un intermédiaire qui constitue, à première vue, une zone plus floue, c'est-à-dire à la fois moins manifeste que les images (elles n'acquièrent de sens qu'à l'analyse) et moins structurée que les catégories symboliques (les termes opposés peuvent par exemple coexister) : bref, une zone qui correspond en gros aux 
représentations telles qu'elles pouvaient être énoncées par des individus différents, dans leur variété et leur diversité, et qui fait référence à l'expérience de la personne interrogée. Tout se passe en effet comme si c'était l'expérience qui permettait d'aller au-delà des images et de faire fonctionner l'ancrage. Nous avons vu comment, à propos des représentations de la chimie, les expériences scolaire, professionnelle, quotidienne, etc. renvoyaient à des modèles, des systèmes de valeurs, des attitudes différents. Rien d'étonnant donc à ce que ce soit à ce niveau, où les représentations prennent sens pour le sujet à partir de son expérience, que l'on voit souvent se définir les attentes dans un "voilà ce que je pense que pourrait être l'exposition ou le musée". Le visiteur n'est plus a priori intéressé par la recherche scientifique, il a ses propres motivations, qui sont nées de sa propre histoire et qui à la fois font le lien avec les systèmes sociaux de valeurs et les catégories symboliques.

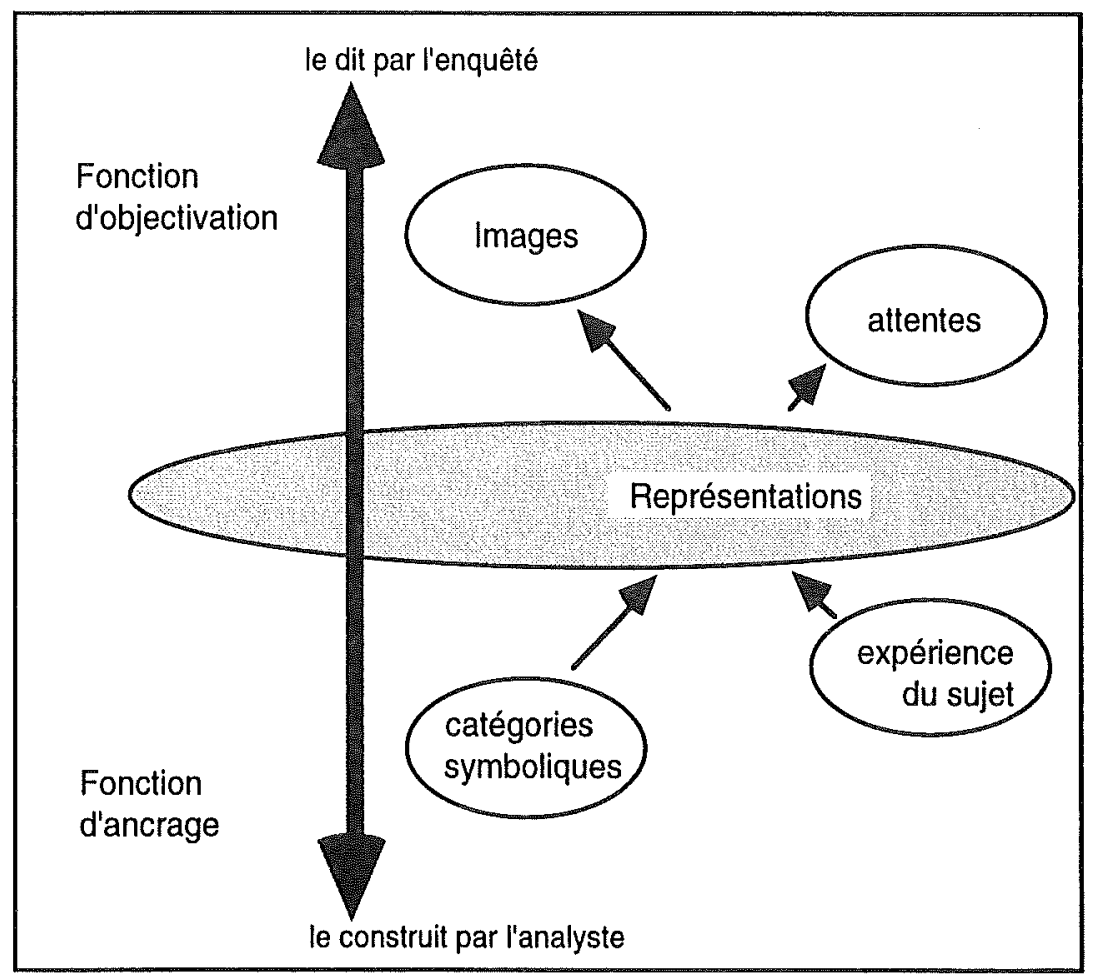

Le système des représentations, entre l'ancrage et l'objectivation 
Le point à retenir ici est qu'à partir de cette analyse des représentations, il est possible de distinguer trois statuts différents du discours des personnes interrogées; ce qui correspond, en fin de compte, à trois prises de position différentes par rapport à ce que peut proposer l'exposition ou le musée.

1. On pourrait être tenté de retenir principalement les images du fait de leur caractère massif et évident. Elles sont proches des stéréotypes, des symboles; elles correspondent à ce qui vient en quelque sorte spontanément à l'esprit lorsqu'on cherche à se représenter l'environnement ou la chimie (par exemple, l'image de l'usine dégageant de la fumée). De ce point de vue, elles ont quelque chose d'une "prise de position" au sens où énoncer une image implique que le sujet qui le fait adhère à elle. Cependant, une telle prise de position est moins revendiquée explicitement qu'adoptée implicitement. D'un point de vue individuel, nous sommes proches de ce que les psychologues définissent comme les attitudes. D'un point de vue pratique, ce fonctionnement des images nous rappelle à quel point l'exposition peut précisément fonctionner comme un lieu de construction de sens par des condensations poétiques de multiples concepts, événements, gestes, façons de faire.

2. Tout à fait à l'opposé, les attentes peuvent paraître n'avoir que peu de valeur, car précisément, elles sont ce que les personnes interrogées disent explicitement demander ou souhaiter vis-à-vis de ce que doit être une exposition, les missions qu'elle doit satisfaire ou les besoins auxquels les personnes souhaitent qu'elle réponde. À travers elles, les futurs visiteurs prennent position par rapport au thème luimême, et par rapport au musée ou à l'exposition. Mais cette prise de position paraît souvent au muséologue infondée, à la fois non légitime (le visiteur se met à la place de celui-ci et s'installe sur un domaine qui n'est pas le sien) et incohérente (les raisons avancées le sont de son seul point de vue d'utilisateur et laissent échapper toute une série de facteurs qui lui échappent). On peut remarquer que les personnes interrogées se sentent mal à l'aise pour affirmer une telle prise de position, soit qu'elles estiment ne pas être fondées à s'avancer ainsi, soit qu'elles aient du mal à se représenter l'objet résultant de leurs attentes ${ }^{1}$. Notons d'ailleurs que les attentes ne viennent souvent qu'en

1 Pour une recherche sur les moyens permettant une formulation des attentes -et plus généralement d'ailleurs des représentations-, voir H. GotTESDIENER et J. DAVALLon, "Le Musée national des Techniques sous l'œil de ses visiteurs", Musée des arts et métiers: la revue, 1, sept. 1992, p. 34-39 et IDEM, "Du visiteur 
fin, lorsque la personne a déjà énoncé la manière dont elle se représente le sujet sur lequel porte l'entretien.

3. Les images et les attentes que le public exprime sont analysées très différemment de ce que permettent les études préalables "classiques", dès lors qu'elles ne constituent qu'un élément des représentations. Car avec l'analyse des représentations, on passe d'un simple recueil de ce que dit la personne à une saisie de ce qui organise sa façon de voir les choses. En un sens, on peut dire que les représentations sont plus profondes (moins explicites) et plus structurantes que les images et les attentes. Cela permet de donner un sens à ces prises de position au regard du travail opéré à partir tant des catégories symboliques que de la mise en perpective tenant au type d'expérience mobilisée.

\section{Ce type d'évaluation conduit à une nouvelle approche du visiteur}

L'entreprise, qui consistait au départ à contribuer à optimiser la conception d'une exposition ou d'un musée, est ensuite venue prendre place dans une réflexion plus théorique sur la manière dont il convenait d'aborder et donc de définir l'exposition aujourd'hui. Nous avons été alors amenés à ouvrir la discussion sur un certain nombre de points qui dépassent largement le cadre non seulement de ces deux études, mais encore de l'utilisation du modèle des représentations sociales par l'évaluation ou bien du traitement de thématiques à forte dimension sociale par le musée de science.

En effet, la manière -dans la théorie comme dans la pratiquedont on pense l'évaluation présuppose une certaine manière de voir et de définir l'exposition. Celle qui est en arrière-plan d'une étude de représentations sociales dans le cadre d'une évaluation préalable est une conception communicationnelle de l'exposition. Avec "l'effet structurant de l'école des communications sur la muséologie", selon l'expression de Bernard Schiele ${ }^{1}$ qui a mis en lumière le lien entre communication, conception et évaluation, on assiste depuis la période après-guerre à un effort de rationalisation de la conception :

«interrogé» au visiteur «expert», in Symposium franco-canadien sur l'évaluation des musées, 8-9 déc. 1994, Québec, Musée de la civilisation, coll. Document, $\mathrm{n}^{\circ} 21$, 1995 , p. 89-93.

1 B. SCHIELE, op. cit. 
planification des étapes de production, multiplication des procédures de contrôle des objectifs et des effets, parmi lesquelles l'évaluation. Cette dernière, initialement dédiée à la démarche éducative qui soustendait la muséologie des sciences et des techniques, peut sans difficulté se prêter aujourd'hui aux enjeux nouveaux d'une gestion de la production d'expositions qui soit la plus efficace possible.

Dans ce contexte, plusieurs usages des études de représentation en tant que moyen de connaître le visiteur (et de le reconnaître comme actif) afin de mieux prévoir les effets de l'exposition sur lui, sont possibles. D'un côté, elles fournissent une approche des "conceptions naïves" (misconceptions) des visiteurs. Leur prise en compte dans l'exposition permet une efficacité plus grande de celle-ci en évitant que ces conceptions naïves ne viennent perturber les savoirs scientifiques, interférer avec eux, les barrer ou se substituer à eux ${ }^{1}$. À l'opposé de cet usage en vue d'une rationalisation didactique de la communication, nous trouvons celui visant la rationalisation médiatique de la communication : la connaissance des représentations (en fait, essentiellement celle des images et des attentes) sert à faire de l'exposition un produit qui satisfasse les visiteurs.

Or, l'étude des représentations sociales telle que nous venons de la discuter se démarque de ces deux approches. Elle définit le visiteur non du point de vue du contenu à assimiler ou du point de vue de ses motivations à consommer, mais comme une entité en soi. La différence porte donc sur la définition même du visiteur. Car, si chaque conception de l'évaluation implique une conception de l'exposition, elle présuppose corrélativement une "psychologie" du visiteur. Pour faire vite: tandis que dans les deux premiers cas, le visiteur est défini en tant que frein potentiel à la transmission du savoir par l'exposition (approche du caractère négatif de son activité) ou en tant que détenteur de la clé de ce que doit être l'exposition (son activité est le résultat d'un intérêt), dans l'approche des représentations sociales, il est considéré comme l'un des deux pôles d'une interaction dont le second est l'exposition. Il n'est plus un récepteur incompétent, ni à l'opposé un consommateur tout-puissant, mais un sujet doté d'une "compétence communicationnelle". Cette dernière est en effet, nous l'avons vu, pensée comme un cadre à la fois affectif, cognitif et

1 Voir, par exemple, M. BorUN, C. MASSEY et T. LUTTER, "Connaissances naïves et conception d'éléments d'exposition dans les musées de sciences", Publics \& musées, 4, mai 1994, p. 27-45. 
social, qui va servir au visiteur pour appréhender ce que lui propose l'exposition. Il est alors normal que l'interaction entre visiteur et exposition soit définie comme une "appropriation" qui vient répondre à une ou des "stratégies communicationnelles"1.

Mais les éléments qui ressortent à la discussion des deux études nous amènent à aller plus loin encore dans la reconnaissance des visiteurs; car ces derniers y prennent, si l'on peut dire, une "épaisseur" plus grande. Et c'est cela même qui appelle réflexion. Non seulement ils y apparaissent comme des sujets dotés d'une compétence communicationnelle plus ou moins en rapport avec l'exposition et plus ou moins adaptée à elle, mais ils y sont des acteurs sociaux développant une opinion, dotés d'une compétence sociale sur un domaine qui se trouve être "aussi" celui dont traite l'exposition. C'est autour de la thématique en tant qu'objet social que se positionnent visiteur et exposition; c'est à cause d'elle qu'ils vont se rencontrer.

\section{Faut-il revoir notre conception de la communication des savoirs par l'exposition?}

Mettre en perspective les diverses modifications que l'étude des représentations sociales introduit dans l'évaluation tant du point de vue des modalités d'analyse, du statut du discours et du visiteur, ou même de l'usage de l'évaluation, fait apparaître une ouverture du système savoir-exposition-visiteur. Faisons le point sur ce que recouvre cette ouverture afin d'essayer d'en appréhender les conséquences sur le fonctionnement communicationnel de l'exposition.

\section{Un élargissement du dispositif communicationnel}

Jusqu'à présent, l'évolution de l'évaluation et de l'exposition nous avait habitués à un déplacement du centre de gravité du système, l'accent étant mis tantôt sur le savoir à transmettre, tantôt sur l'exposition comme moyen, tantôt sur le visiteur comme destinataire. Le

1 B. SCHIELE, "Notes pour une analyse de la compétence communicationnelle de l'exposition scientifique", Loisir et société/Society and Leisure, 10 (1), print. 1987, p. 45-67 et J. Davallon, "Peut-on parler d'une «langue» de l'exposition scientifique", in B. SCHIELE (éd.), Faire voir, faire savoir: la muséologie scientifique au présent, Actes du colloque international, 18 oct. 1989, Montréal, Québec, Musée de la civilisation, 1989, p. 47-59. 
problème majeur auquel devait aider à répondre l'évaluation était la maîtrise des relations entre les trois sous-systèmes pour optimiser le dispositif de communication du savoir au visiteur par l'exposition, sans sortir pour autant d'un système abordé comme un dispositif fermé. Ce qui retient l'attention dans le cas que nous venons d'examiner, c'est que le système se retrouve en quelque sorte plongé dans son environnement social. Nous n'avons plus affaire à un dispositif communicationnel restreint organisé autour de la prise d'information par le visiteur et de sa compréhension du contenu scientifique présenté, mais plutôt à un dispositif élargi. Qu'entendre par là ?

Retournons-nous sur le chemin parcouru depuis le début de cet article. Trois marques d'élargissement du dispositif communicationnel se détachent clairement.

1. La plus évidente (et la plus proche de notre point d'arrivée) touche la définition du visiteur. Reconnaître les visiteurs comme des acteurs sociaux et non seulement comme les récepteurs d'une information ou comme les consommateurs d'un produit, revient à prendre acte du fait que ces acteurs sociaux ont été constitués en dehors de l'exposition. Ils ont pu l'être en tant que visiteurs de musées de science (ou d'autres musées), mais aussi en tant que lecteurs de revues ou de journaux, que spectateurs de télévision, qu'usagers de l'école, etc. Et que fait alors l'étude de représentations sociales sinon de faire apparaître, d'énoncer et de rendre visible le résultat de cette constitution du visiteur ? Elle rend public le résultat d'un processus de socialisation; elle le "publicise" en lui offrant un lieu et une forme d'expression, c'est-à-dire en le faisant exister sur une scène publique. L'enquête présente des pratiques, des opinions, des prises de positions, des manières de faire dont la diversité même contribue à construire la "figure" du public. Ce faisant, on peut dire qu'elle "représente" le public puisque les pratiques et opinions concrètes énoncées dessinent des rôles, des goûts, une forme à partir de laquelle des individus réels pourront en retour agir et penser ${ }^{1}$. Elle le représente donc au sens où elle instaure une médiation qui extériorise des opinions, des idées individuelles, et comme tel fournit un cadre -une forme, un langage, des référents; bref un lieu commun- permettant un

1 Hélène Merlin (Public et littérature en France au XVII ${ }^{e}$ siècle, Paris, Éd. Les belles lettres, coll., Histoire, 1994) définit très heureusement le public comme "un être idéel fait d'individus réels qui se pensent dans cette forme"; cet être idéel n'est ni origine, ni résultat des idées, il est une "forme", une "configuration de rôle", "une dramaturgie". 
positionnement, une discussion, un échange entre des gens intéressés à des titres divers par ce dont elle traite' ${ }^{1}$. Non seulement l'étude des représentations sociales enrichit la représentation que l'on se fait des visiteurs, mais elle représente le public en ce qu'elle donne la possibilité à des acteurs sociaux de former et d'exprimer une opinion qui les rend présents en tant que public. S'il y a représentation, c'est en un sens assez proche de la représentation du peuple en politique ou du public par la critique ${ }^{2}$.

2. La seconde marque d'élargissement du dispositif communicationnel concerne le savoir qui n'est plus seulement considéré comme un contenu scientifique, mais comme un objet social. Nous avons déjà parlé à plusieurs reprises de cette modification du statut du savoir. Il demanderait certes à être vérifié et précisé, d'autant plus qu'il renvoie à nombre de discussions concernant la diffusion des sciences. D'aucuns ne manqueront pas en effet de se demander si une telle approche sociale du savoir scientifique ne revient pas remplacer celuici par l'opinion que l'on a sur lui. En réalité, ce type de crainte n'a de sens que dans le cadre du dispositif communicationnel restreint. C'est à ce niveau que se pose la nécessité d'un contrôle de la compréhension par le visiteur afin que celle-ci soit conforme au contenu et à la

1 L. QuÉRÉ, "D'un modèle épistémologique de la communication à un modèle praxéologique", Réseaux: communication, technologie, société, 46-47, mai-juin 1991, p. 69-90.

2 L'évaluation est porte-parole d'un jugement des visiteurs mobilisant des savoirs sociaux, au sein d'un espace où la critique n'existe pas. L'évaluation développe alors institutionnellement la possibilité d'un discours du visiteur. Une analyse des procédures de cette représentation du visiteur dans le discours de l'enquête fait l'objet de la thèse en cours d'achèvement de Joëlle Le Marec. Pour une première approche, voir J. LE MAREC, "Méthodes d'accès au savoir dans l'exposition scientifique: anticipation des intentions et représentations des visiteurs" in B. SCHIELE, Quand la science se fait culture, Actes II, Colloque international, Montréal, 10-13 avril 1994, Sainte-Foy (Québec), Éd. MultiMondes, 1994 (2 disquettes). La question de l'importance de la représentation des usagers des médias est soulevée par P. Chambat, "NTIC et représentation des usagers", in A. VITALIS (éd.), Médias et nouvelles technologies: pour une socio-politique des usages, Paris, Ed. Apogée, coll. Médias et nouvelles technologies, 1994, p. 125). Dans le cas des expositions, nous avons essayé de discuter les relations entre évaluation et critique, opposant l'usage fonctionnel qui est généralement fait de la première à l'absence (ou la timide émergence) d'un espace public allant de pair avec la seconde dans Davallon et Le Marec (à par.), "Questions posées pour une approche symbolique des musées à l'évaluation", in Symposium franco-canadien sur l'évaluation des musées, Actes du symposium tenu les 22-24 mars 1995 au Centre Georges Pompidou, Dijon, Office de coopération et d'information muséographiques. 
logique du savoir scientifique. Au niveau du dispositif élargi, la question du statut social du savoir scientifique se pose en d'autres termes. Du côté des visiteurs (qui est le côté que nous avons exploré), lorsque les savoirs scientifiques mobilisés portent sur des sujets constituant un enjeu social et politique, comme c'est le cas pour l'environnement, cette question prend la forme d'une interrogation sur la crédibilité et la fiabilité de l'information délivrée par le musée et, en comparaison, par les autres médias. C'est dire que loin d'éliminer la logique du savoir scientifique (et on pourrait ajouter la démarche scientifique), l'attention portée au statut social de ce savoir vise au contraire à s'assurer que les contenus présentés peuvent permettre de se faire une opinion sur l'enjeu social et politique. La question porte donc sur la crédibilité des opérations de médiation effectuées par les médiateurs. Elle se situe sur le plan de la relation entre le visiteur et le musée (pris en tant qu'instance énonciatrice) et sur le plan de leur engagement de l'un vis-à-vis de l'autre et non seulement sur celui d'une conformité de l'information. Ou plus exactement, cette conformité fait partie de l'engagement.

Le troisième élargissement concerne la forme et le fonctionnement du dispositif communicationnel lui-même, c'est-à-dire de l'exposition en tant que médiation des savoirs scientifiques et techniques'. C'est par l'examen de ce point que nous voudrions terminer.

1 Nous distinguons la médiation de la médiatisation. L'organisation formelle de l'exposition comme média est une médiatisation, c'est-à-dire qu'elle régit, selon la technologie de la mise en exposition (et les règles du genre "exposition"), d'une part la relation du visiteur à l'exposition-objet (et au-delà, à l'instance productrice de cette dernière) et d'autre part la relation du visiteur au message de l'exposition (la production de sens attendue du visiteur). Cette médiatisation introduit une médiation entre le monde du visiteur et celui de la science. La médiatisation est un phénomène médiatique (i.e. lié au fonctionnement du média), la médiation un phénomène symbolique, anthropologique (i.e. lié à la fonction sociale du média). Les sociologues ont considérablement rénové l'approche de la médiation ces dernières années soit sous la catégorie de la traduction (v.g. B. LATOUR, "Quand les anges deviennent de bien mauvais messagers", Terrain: carnet du patrimoine ethnologique, 14, 1990, p. 76-91), soit directement sous une étude des médiations (A. Hennion, La passion musicale. Une sociologie de la médiation, Paris, Éd. Métailié, coll. Leçons de choses, 1993). Avec notre conception du dispositif communicationnel élargi, nous sommes en phase avec une grande part des analyses développées par Antoine Hennion. 


\section{Pour aborder l'exposition comme une situation de communication}

Que faut-il précisément entendre par élargissement du dispositif communicationnel de l'exposition? Pour répondre, revenons sur le traitement du savoir par l'exposition.

L'exposition, en tant que dispositif de diffusion des savoirs scientifiques ou techniques, opère une "publicisation" de ces savoirs. Cela signifie qu'elle présente ces savoirs en disposant dans l'espace et en articulant en discours (l'un et l'autre, l'un par l'autre) des outils représentant ces savoirs: objets, schémas, discours, manipulations, reconstitutions, etc. ${ }^{1}$. Habituellement, on retient les déformations que cette double opération de représentation (représentation de la mise en scène et représentation d'un savoir par des délégués) entraîne sur les discours scientifiques et la nécessité de contrôler les effets produits; concepteurs et évaluateurs se trouvant alors engagés dans une quête de transparence de la représentation. Or, la réflexion que nous venons de proposer invite à mettre l'accent sur un autre aspect de cette représentation; à savoir, sur le fait qu'elle ouvre une nouvelle scène pour le savoir scientifique, une nouvelle scène sur laquelle le rendre public. Pour dire les choses autrement : la transformation qu'opère la représentation introduit les savoirs ainsi "interprétés" sur une autre scène sociale que celle où ils ont été produits et, nous l'avons dit, change son statut social. Elle les extrait du cercle restreint de la communication intérieure au monde scientifique; elle les sort aussi du domaine habituel de la vulgarisation des sciences qui reste dominé par le discours linguistique et offre ainsi aux visiteurs des modalités de rencontre nouvelles dont les caractéristiques sont liées à la spécificité du média-exposition. Par conséquent, la "publicisation" des savoirs par l'exposition (ou le musée) n'est pas à penser comme le simple prolongement d'un processus de diffusion des savoirs déjà existant; elle est à concevoir comme l'introduction d'un nouveau média dans un champ -avec tout ce que cela suppose d'effets sur le champ luimême, mais aussi sur le média en question. C'est alors l'image d'un processus de diffusion des savoirs considéré comme monolithique, unitaire depuis sa source jusqu'à son point d'arrivée et abordé de surcrôt du seul point de vue des avatars qu'il fait subir à ces savoirs

1 J. Davallon, "Exposition scientifique, espace et ostension", Protée, 16 (3), sept. 1988, Chicoutimi, Université du Québec à Chicoutimi, 1988, p. 5-16. 
au cours de leur transmission qui doit être abandonnée au profit d'une analyse des relations et des effets réciproques entre ce nouveau média et le champ de la médiation des savoirs scientifiques et techniques. Se pencher sur l'élargissement du dispositif communicationnel de l'exposition est un moyen de contribuer à une telle analyse en partant de la forme et du fonctionnement de l'exposition.

Le changement de point de vue, impliqué par un regard porté sur la manière dont l'exposition s'insère et se développe dans le champ de la médiation des savoirs, conduit en effet à porter attention à certaines des modifications du dispositif communicationnel de l'exposition.

Nous avons vu que l'enquête des représentations sociales opérait une représentation de l'intérêt des visiteurs, en tant qu'acteurs sociaux, pour le savoir scientifique. Le fait que cette représentation puisse passer (au moins partiellement) dans la conception même de l'exposition à la faveur de l'utilisation des études préalables par les concepteurs et faire au bout du compte que l'exposition représente cet intérêt ${ }^{1}$, attire l'attention sur le fait que l'exposition est au centre d'un réseau d'intérêts croisés venant des visiteurs mais aussi des scientifiques, des médiateurs. Des intérêts qui, au moins dans le cas des visiteurs, portent à la fois sur le savoir scientifique et sur le média luimême. Pour dire les choses autrement : on va visiter une exposition pour ce dont elle traite, mais on découvre ce qu'elle traite dans le plaisir de la visiter. La sociologie des médiations ${ }^{2}$ nous a d'ailleurs fort opportunément rappelé -après la théorie de la représentation d'un Louis Marin par exemple- l'importance de cet intérêt porté aux médiations non seulement dans celui que l'on porte à ce qu'elle traite, mais aussi dans le développement et l'institutionnalisation de ces médiations. Le développement de l'exposition doit tout autant au plaisir de l'exposition qu'à la nécessité de diffuser les savoirs.

Le point à retenir ici est que tout cela -c'est-à-dire le fait que l'exposition soit au centre d'intérêts et qu'elle soit un média appréciépousse au développement de ce nouveau média au côté de ceux déjà existants (comme la presse) ou de ceux émergeants (la télévision ou les nouvelles techniques de communications). On voit alors à quel point ce développement de l'exposition est lié aux deux autres marques d'élargissement du dispositif communicationnel: la constitution d'une compétence sociale d'un visiteur "amateur de

\footnotetext{
1 J. LE MAREC, "Les évaluations préalables...", op. cit.

2 Br. LATOUR, op. cit. ; A. HENNION, op, cit.
} 
science" et l'émergence d'une approche des savoirs comme objets sociaux. L'exposition, en entrant en interaction avec ce visiteur et cette approche, change son mode de médiation des savoirs.

En effet, tant que le dispositif communicationnel est restreint et répond au modèle d'une communication conçue comme transmission d'un savoir, la médiation s'exerce sur le mode d'une autorité interprétante garante de la bonne production du sens en bout de chaîne'. Lorsque le dispositif s'élargit et que l'exposition entre en interaction avec un visiteur fonctionnant comme un acteur social indépendant ayant (ou cherchant) un point de vue sur le savoir exposé, la production de sens chez le visiteur n'est plus que partiellement directement contrôlable. Le recueil du discours des visiteurs devient de première utilité, puisqu'il est le moyen d'évaluer l'effet produit; d'où le développement de l'évaluation. Cependant, comme cet effet n'est plus produit seulement par l'exposition elle-même, mais qu'il est la résultante des interactions se déroulant au sein du champ de la médiation des savoirs dans son ensemble et impliquant à la fois divers médias, différents acteurs et plusieurs types de savoirs traités, l'hégémonie de l'autorité interprétante est remplacée par un jeu de représentation (de savoirs, d'intérêts, de choses, etc.) plus ou moins médiatisée et d'interprétation par les acteurs. La production de sens sera alors au croisement de la visite et de lectures, le traitement du même sujet par d'autres médias, de commentaires et de discussions, etc. On voit comment en changeant de niveau, nous passons du système (relativement) fermé du dispositif communicationnel de l'exposition vers le système ouvert d'un dispositif élargi qui se déploie dans un espace social incluant l'exposition, mais aussi d'autres médias.

Est-ce à dire que cette mise en réseau revient à une dilution de l'exposition et à une production de sens par le visiteur qui soit aléatoire? On pourrait le craindre compte tenu du fait que le développement de l'exposition comme média introduit une (ou des) médiation(s) supplémentaire(s) qui soit entre(nt) en résonance avec d'autres, soit vien(nen)t s'ajouter à elles. L'ouverture du système va en effet de pair avec un allongement et une complexification du trajet qui va du texte-source (appartenant au discours scientifique) au destinataire puisqu'il peut passer alternativement et successivement par divers

1 Le contrôle de cette autorité est évidemment un enjeu social de première importance, d'où les discussions sur qui est autorisé à exercer la médiation, des scientifiques ou des médiateurs. 
médias du fait de leur diversification, de leur empilement ou de leur emboîtement. La réponse est double, car deux phénomènes sont à prendre en compte. D'un côté, on peut penser que la diversification et la complexification du champ de la médiation des sciences et des techniques ne signifie pas ipso facto sa déstructuration, mais plutôt sa transformation. Mais il s'agit-là d'un domaine de recherche qui dépasse largement le seul média exposition et inclut par exemple l'étude de l'usage des nouvelles technologies de communication à des fins de médiation des savoirs. D'un autre côté, l'élargissement du dispositif communicationnel de l'exposition ne signifie pas sa disparition. Le dispositif de l'exposition continue d'exister.

En effet, le changement suggéré par notre réflexion ne porte pas sur le devenir de l'exposition mais se situe du côté de la manière dont on analyse celle-ci. L'exposition était considérée comme un instrument, comme un outil de communication; son approche tentait donc de s'aligner sur une conception de la communication comme transmission et une définition technologique et fonctionnelle des médias. Elle nous apparaît ici sous l'autre versant du média, moins comme un dispositif de médiatisation que comme une forme de médiation. Elle serait par conséquent à aborder comme un processus plus que comme un objet: comme l'instauration d'une situation de communication entre des acteurs et des savoirs.

Loin de disparaître, l'exposition semble plutôt se retrouver aujourd'hui au centre d'un espace social, développant un public et relayée par d'autres médias. La diversification des activités des musées de sciences et techniques paraît la marque d'une stratégie correspondant à cette évolution. 